\title{
Effects of chemotherapy on ultrastructure of oesophageal adenocarcinoma
}

\author{
S J Darnton, G N Antonakopoulos, J Newman, H R Matthews
}

\begin{abstract}
Aims: To compare and contrast the ultrastructural appearance of resected oesophageal adenocarcinomas treated with preoperative chemotherapy with that of non-treated resected controls; and to determine the usefulness of this method in the assessment of the effectiveness of the chemotherapeutic regimen.

Methods: Ten resected oesophageal adenocarcinomas treated with preoperative chemotherapy-mitomycin-C, ifosfamide, and cisplatin (MIC)-were examined by transmission electron microscopy and their appearance compared with that of 13 concurrent untreated resected oesophageal adenocarcinomas.

Results: The treated adenocarcinomas showed cytotoxic damage although complete tumour eradication was not achieved. In all 10 treated cases a variable proportion of the neoplastic cells showed unusual degenerative and necrotic changes not seen in untreated cases. In the most affected carcinomas the stroma contained increased numbers of inflammatory cells.

Conclusions: This ultrastructural method is useful for the assessment of the in vivo effect of MIC.
\end{abstract}

(F Clin Pathol 1992;45:979-983)

Adenocarcinoma of the oesophagus or oesophagogastric junction is rare but rapidly fatal. ${ }^{1}$ At this hospital neoadjuvant combination chemotherapy (mitomycin- $\mathrm{C}$, ifosfamide, and cisplatin or MIC) was used before resection of primary oesophageal adenocarcinoma in a Phase II study. The clinical details have been published elsewhere. ${ }^{2}$

Oesophageal Research

Laboratory

S J Darnton

Department of

Histopathology

G N Antonakopoulos

J Newman

Department of

Thoracic Surgery

H R Matthews

East Birmingham Hospital, Birmingham B9 5ST

Correspondence to: Dr S J Darnton

Accepted for publication 14 May 1992

\section{Methods}

Between December 1989 and July 199123 consecutive cases of localised adenocarcinoma of the lower oesophagus or oesophagogastric junction were treated by resection with or without preoperative chemotherapy.

Ten patients (nine men, mean age 58.7 years, range 38-68) received chemotherapy (MIC). Of these, nine patients received two preoperative pulses with an interval of three weeks, followed by resection three weeks later. ${ }^{2}$ One additional man aged 70 years received only one pulse of MIC three weeks before resection. Thirteen patients (nine men, mean age $64 \cdot 2$ years, range $50-73$ ) received no neoadjuvant chemotherapy.

Immediately after resection each specimen was opened longitudinally and pinned out. Photography, mapping, and routine microscopic examination were performed. A fresh blade was used to take a cube of tumour (of about $5 \mathrm{~mm}$ size) and also a strip (about $5 \times 2 \mathrm{~mm}$ ) of "normal" oesophageal mucosa from a proximal site on the specimen, as far away from the tumour area as possible. The samples were placed in cold buffered glutaraldehyde, $\mathrm{pH} 7 \cdot 4$.

After fixation in glutaraldehyde, the samples were cut into $2 \mathrm{~mm}$ size cubes. Special care was taken to select the most viable looking portions which were then post-fixed in $1 \%$ osmium tetroxide in phosphate buffer $(\mathrm{pH}$ 7.4), dehydrated in ethyl alcohol, and after treatment with inhibisol embedded in TAAB resin. All stages were prolonged to avoid possible poor embedding because of the unusually large size of the samples. Thick sections were stained by toluidine blue and examined by light microscopy, while thin sections were stained by uranyl acetate and lead citrate and examined in a JEOL JEM-100C transmission electron microscope.

In one of the cases treated with MIC immunohistochemistry for PGP 9.5 (Protein Gene Product 9.5, UltraClone Ltd., Rossiter's Farmhouse, Wellan, Isle of Wight PO41 OTE) was performed on the paraffin wax embedded tissue to confirm the presence of neuroendocrine cells.

\section{Results}

The appearance of oesophageal squamous epithelium taken from an area distant from the tumours of treated and untreated cases showed no obvious differences within the three defined layers-basal, prickle, and functional cells.

The appearance of the untreated group of adenocarcinomas was identical with that which we have described in our previous study.

All 10 cases treated with neoadjuvant MIC showed features not seen in the untreated cases. These features were restricted to the appearance of the neoplastic cells and to the number and appearance of inflammatory cells in the intervening stroma. Smooth muscle cells and small vessels generally had an unaffected, conventional appearance. In one case the tumour showed heterogeneous differentiation in that it also contained cells of a neuroendocrine nature. Neurosecretory granules were confirmed on a paraffin wax section by the 


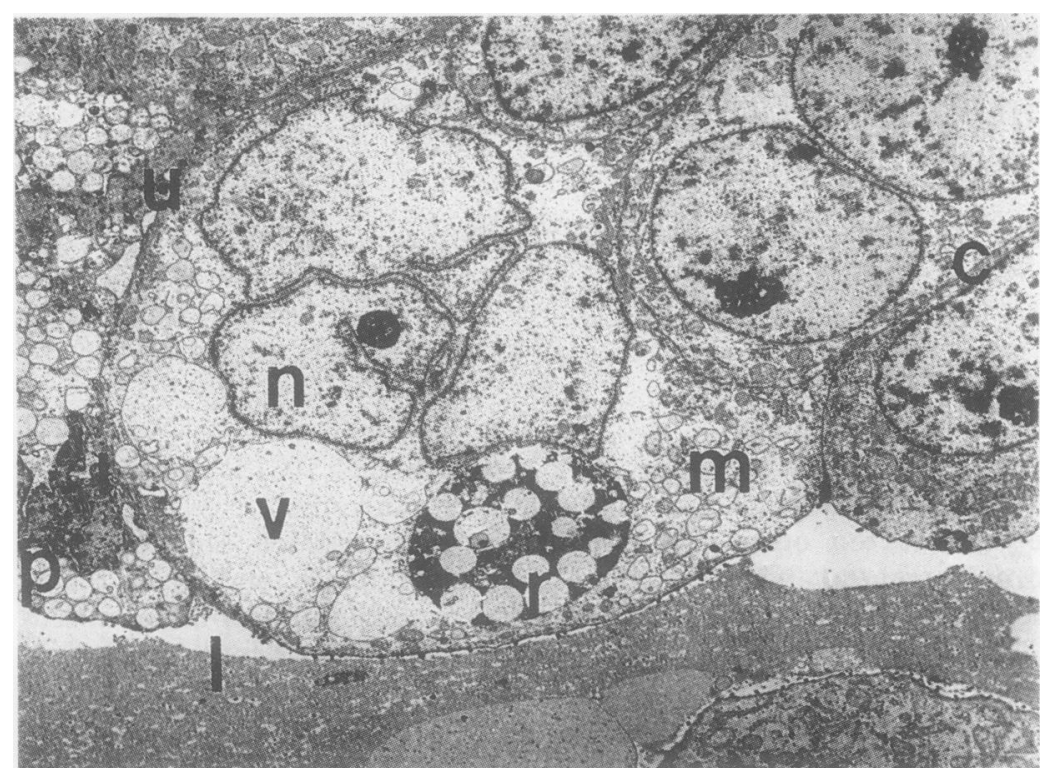

Figure 1 Adjacent conventional cells (c) and unusual cells $(u)$ against a lumen ( $)$. Hypersegmented $(n)$ and pyknotic $(p)$ nuclei. Vacuolated cytoplasm $(v)$ with degenerate mitochondria $(m)$ and a residual body $(r)$.

immunostain PGP 9.5. The neuroendocrine cells had a conventional ultrastructural appearance, although the adjacent neoplastic glandular cells were grossly abnormal. In the same case some desmosomes were seen, a characteristic of squamous rather than glandular differentiation, but tonofilaments were not present.

The 10 treated cases can be divided into two groups on the basis of the fine structure of their tumours. The first group of five cases had a mixed appearance. Cells with conventional, usual features seen in adenocarcinoma were admixed with cells displaying features previously not seen in untreated adenocarcinomas. In the second group of five cases most of the neoplastic cells displayed unusual features.

Group 1: five cases with a proportion of neoplastic cells showing unusual features Many of the tumour cells displayed conven-

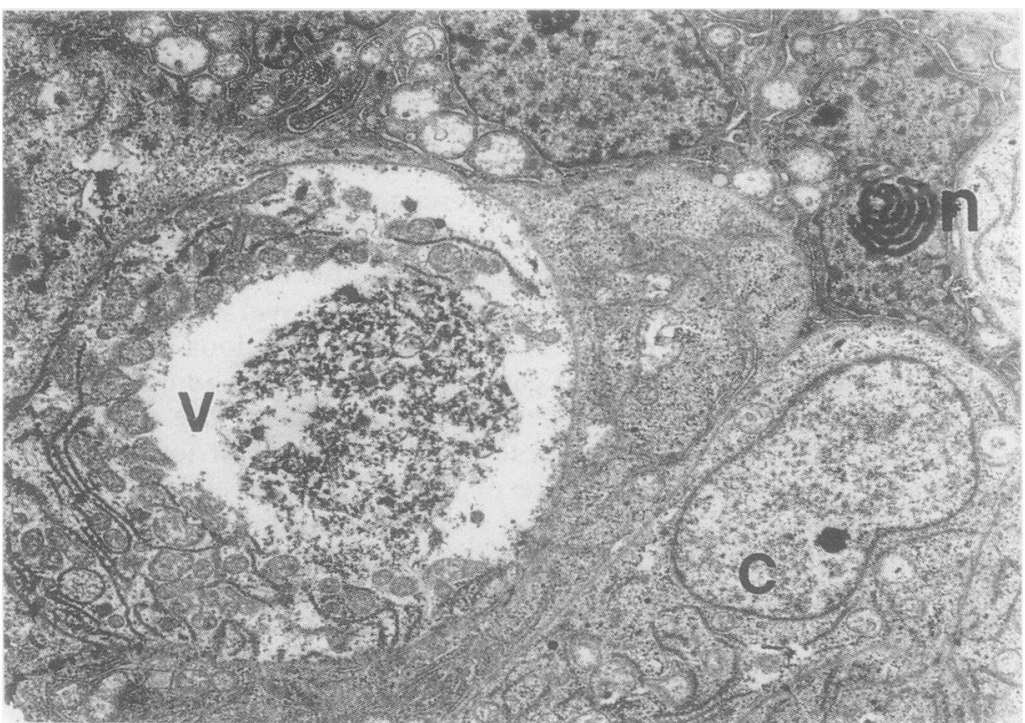

Figure 2 Adjacent conventional cell (c) and unusual cells. An autophagic vacuole (v) contains nuclear material. $A$ nucleolus has an abnormal configuration (n). tional neoplastic appearances seen in adenocarcinomas before. Their nuclei were oval or slightly indented with finely dispersed chromatin and prominent nucleoli. In a few cells the condensed chromatin was marginated with reticulate material in the centre of the nucleus. There was abundant cytoplasm rich in organelles, especially rough endoplasmic reticulum (RER).

A variable proportion of cells showed unusual features. This variability was observed not only among cases but also within the tumour of any individual case. These cells were arranged both in clusters as well as being dispersed between the conventional neoplastic cells (figs 1 and 2 ).

The unusual cells were characterised by misshapen nucleoli (fig 3). In others, the nuclear chromatin was marginated while the more central areas were occupied by a granular material against a background of low electron density. These changes gave the appearance of nuclear "oedema". Occasional whole nuclei were hypersegmented (fig 1). Karyopyknosis and karyorrhexis were seen occasionally.

In the cytoplasm of several of these neoplastic cells were large secondary lysosomes (possibly representing residual bodies (fig l) and autophagic material (fig 3) or secondary necrosis of ingested apoptotic bodies). In all cases were cells with large cytoplasmic vacuoles which sometimes impinged on the indented nuclei (fig 1).

In well differentiated areas of the tumours the most impressive change, seen in all cases, was the protrusion of finely granular cytoplasmic blebs into the lumen. Many appeared to be in the process of being discharged (figs $4 A$ and $B$ ). This feature was consistent with the loss of cellular architecture rather than exocrine secretion, as microvilli, mucin droplets and, in one case, a centriole, were seen within the granular material of these blebs (fig 4B).

In all cases the stroma and tumour clusters contained slightly increased numbers of inflammatory cells including macrophages, lymphocytes, eosinophils and mast cells. Neutrophils were present in small numbers. Several macrophages were seen engulfing amorphous material in the stroma or at the periphery of tumour clusters. These five cases included the one treated with only one pulse of MIC.

Group 2: five cases in which most neoplastic cells displayed unusual features

Most of the tumour cells showed unusual cellular features, although there was some variation within tumours.

The nuclei and their nucleoli were usually irregular in shape and the chromatin was condensed. Karyopyknosis and karyorrhexis were also seen. Convoluted pyknotic nuclei were sometimes located against the basal plasma membrane (fig 5). Rupture of the nuclear envelope with discharge of the nuclear contents into the expanded cisternal network was also seen (fig 6).

Cytoplasmic organisation was disrupted in all cases. Many cells contained large vacuoles and plentiful residual bodies filled with elec- 
Figure 3 Grossly abnormal nucleoli from two unusual cells.
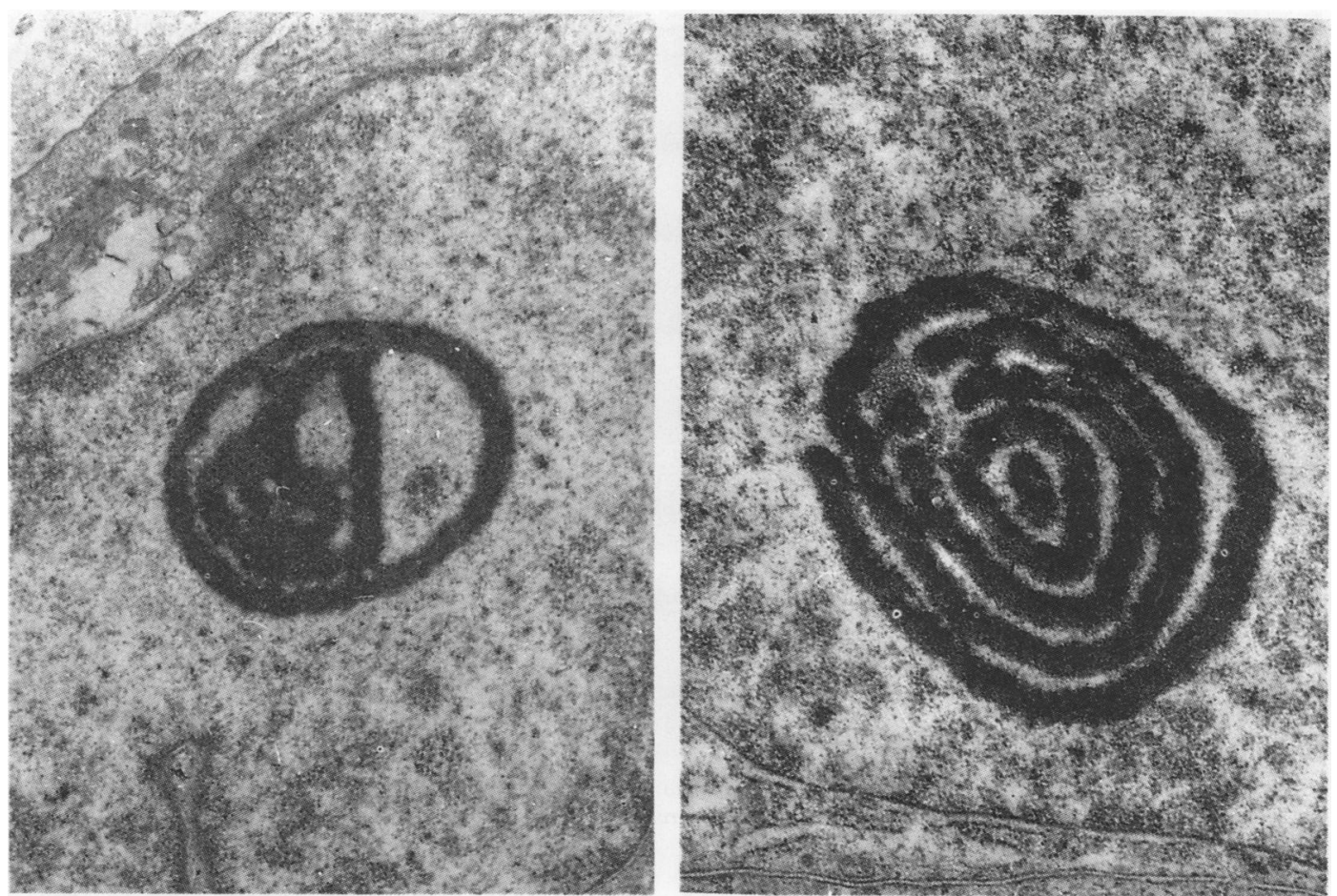

tron dense material. All cases showed apical blebs of granular material. In one case the blebs were so large that they occupied almost the entire lumen of an acinus. Rupture of the cytoplasmic membrane and discharge of cell contents into the stroma was common. Cytoplasmic organelles were disrupted with degenerate mitochondria containing disintegrated cristae and a matrix of low electron density.

The stroma contained abundant macrophages loaded with lipid droplets and electrondense material. These macrophages were seen actively phagocytosing diverse cellular remnants (fig 7). Large numbers of lymphocytes, eosinophils, and mast cells, as well as a few neutrophils, were also present. In all cases many inflammatory cells, especially macrophages and lymphocytes, infiltrated the tumour clusters (fig 8). Abundant active fibroblasts were also present.

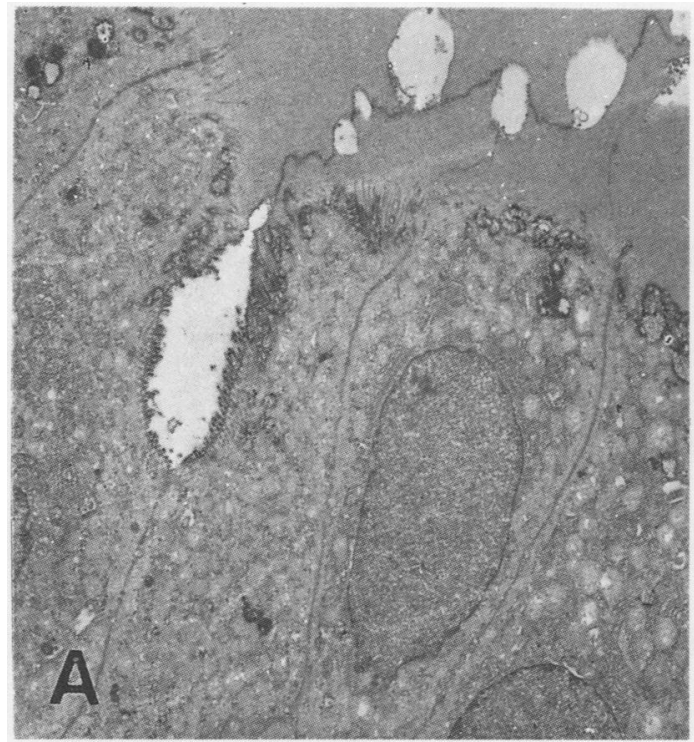

\section{Discussion}

Assessment of the response of oesophageal carcinomas to chemotherapy has been made at this hospital by barium meal, computed tomography, and light microscopy. ${ }^{34}$ Electron microscopic assessment adds another dimension to observations of changes brought about by chemotherapy.

An earlier study of the morphology of oesophageal carcinomas ${ }^{5}$ reported the heterogeneous appearance of adenocarcinomas of the oesophagus. In our study samples from areas of the tumours of treated and untreated cases were selected which included both viable cells and those showing signs of cell death. The treated tumours all showed some signs of the cytotoxic effects of MIC and could be divided into two groups depending on the proportion of tumour cells showing unusual features. The observation that the case given only one pulse

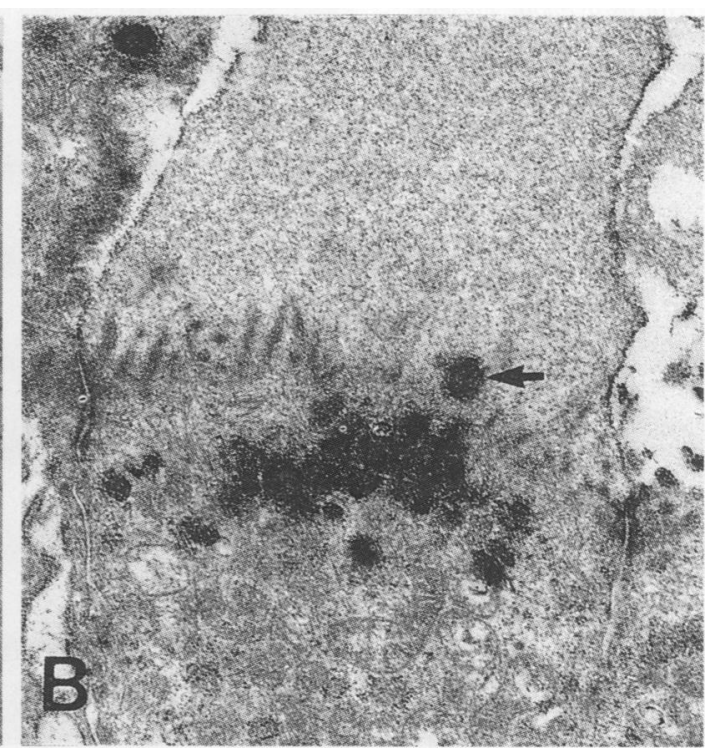

Figure 4 Unusual cells. (A) Apical blebs discharging into the lumen. (B) Apical bleb containing cytoplasmic contents, including a centriole (arrowed). 


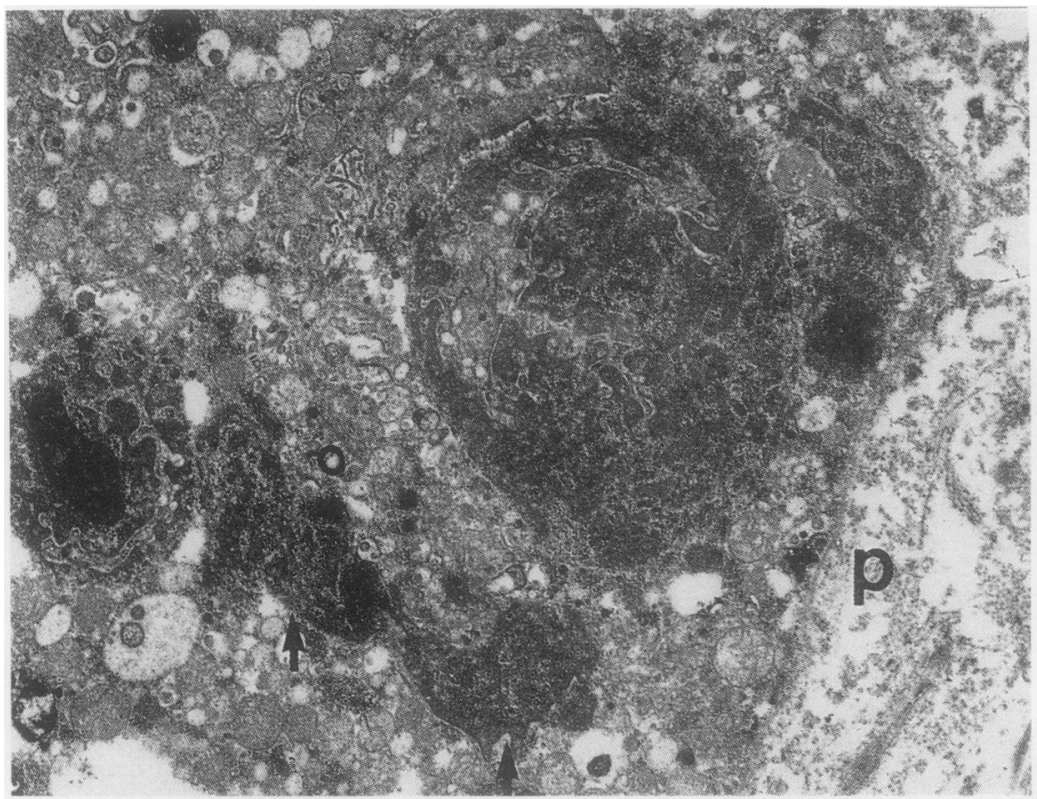

Figure 5 Unusual cell with its pyknotic nucleus adjacent to the basal plasma membrane (p). Condensed chromatin (arrows) is released via a ruptured nuclear envelope.

of MIC fell within the group with the lower level of response may possibly be relevant because the changes seen in that individual case were similar to those in four other cases to whom two pulses were administered but numbers are too small to draw any definite conclusion. Variation occurred within all treated tumours.

The only previous investigation of the ultrastructural effects of chemotherapy on oesophageal tumours was on 14 patients with squamous cell carcinoma. ${ }^{6}$ Various regimens were used and ultrastructural changes were described in only one case, and those were restricted only to nuclear changes-that is, condensation of nucleoli, disintegration of the nucleonema, and reduced electron density of the nucleoplasm with dispersion of chromatin. We have seen similar nuclear changes in the adenocarcinomas treated with MIC, but hypersegmentation of nuclei, abnormally configured nucleoli, and breakdown of the nuclear membrane with resultant spillage of nuclear contents have also been observed.

Detailed examination has shown other signs of cell disruption, presumably due to the cytotoxic effects of the regimen, because these abnormalities were not seen in untreated tumours. There was a complete disruption of cytoplasmic organisation and of the organelles. The location of pyknotic nuclei against the basal plasma membrane of cells suggests collapse of their cytoskeleton.

Similar signs of cell death have been briefly described in two cases of transitional cell carcinoma of the human bladder treated with intravesical mitomycin and studied by biopsy ${ }^{7}$ and of resected normal rat bladder. ${ }^{8}$ Ultrastructural results of in vivo administration of cytotoxins before resection of human carcinomas have rarely been reported, ${ }^{6}$ though similar ultrastructural effects of cytotoxins on human carcinomas transplanted into nude mice have. ${ }^{9}$

The cytotoxic effects of MIC were restricted specifically to the neoplastic cells of the oesophagus, the normal squamous epithelium being totally unaffected (displaying features identical with those previously reported ${ }^{10}$ ). Adjacent smooth muscle and endothelial cells had a normal appearance. The necrotic changes within the tumour cells cannot, therefore, have been secondary to local tissue ischaemia. The retention of a normal local vasculature would be of great therapeutic importance. Any damage to the vasculature would impede delivery of cytotoxins to the target cells.

Heterogeneity was seen in one case out of the 23 in the present series. This case showed neuroendocrine-type cells, unaffected by the chemotherapy, although adjacent glandular cells were grossly abnormal. Such heterogeneity may further complicate successful treatment by chemotherapeutic regimens. Interand intratumour variability emphasises the fact that adenocarcinomas do not behave in a
Figure 6 Unusual cell with its pyknotic nucleus adjacent to the basal plasma membrane (p). Nuclear contents (arrows) spill over into a dilated cisterna of RER

(arrowhead to ribosomes).

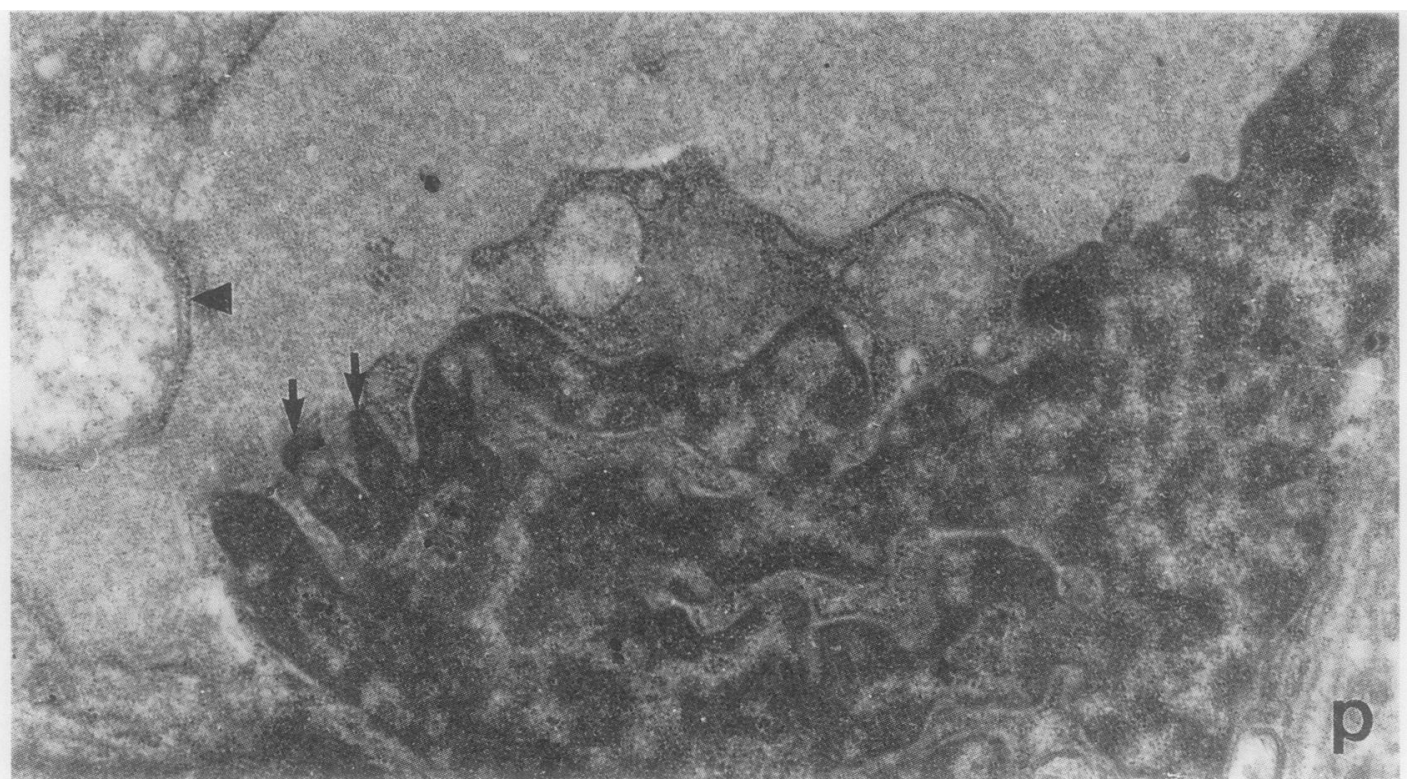


Figure 7 From a disrupted tumour cluster. $A$ lymphocyte (l) adjacent to a macrophage engulfing amorphous material (a).
Figure 8 Lymphocytes () and a macrophage $(\mathrm{m})$ infiltrating a disrupted cluster of neoplastic cells.

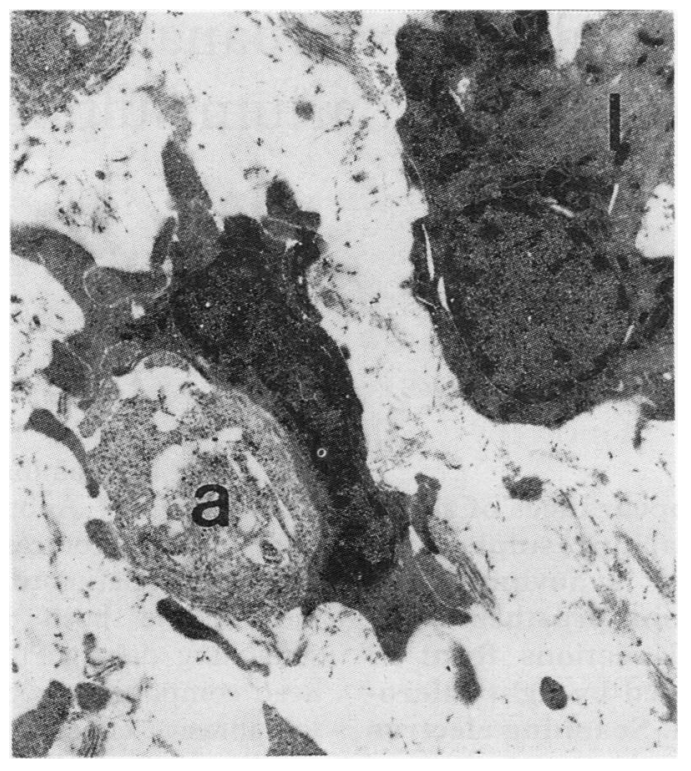

impossible to comment on the level of differentiation of damaged cells, but individual viable cells were of varying degrees of differentiation.

SJD and GNA are supported by the Oesophageal Cancer Research Appeal (OCRA), Birmingham, England. We are grateful to Miss K H Field for photographic assistance.

1 Matthews HR, Waterhouse JAH, Powell J, McConkey CC, Robertson JE, eds. Clinical cancer monographs. Vol 1. Cancer of the oesophagus. London: The Macmillan Press Ltd., 1987:68-7

2. Allen SM, Duffy JP, Darnton SJ, Cullen MH, Matthews HR. A phase II study of mitomycin, ifosfamide and cisplatin in operable adenocarcinoma of the oesophagus. $\mathrm{Br} \mathcal{F}$ Cancer (in press)

3 Walker SI, Allen SM, Steel A, Cullen MH, Matthews HR. Assessment of the response to chemotherapy in oesophageal cancer. Eur f Cardiothorac Surg 1991;5:519-22.

4 Darnton SJ, Allen SM, Edwards CW, Matthews HR. Histopathological findings in oesophageal carcinoma with Histopathological findings in oesophageal carcinoma with and without

uniform manner.

Stromal changes were also seen after treatment. Necrosis of tumour cells presumably recruits host inflammatory responses as immune cells were seen infiltrating the tumours, macrophages engulfed varied material and active fibroblasts were present. These observations agree with the light microscopic changes seen in an earlier study. ${ }^{4}$ It was suggested that the response of oesophageal squamous cell carcinoma to chemotherapy could be seen in the shrinkage of tumour clusters and an increase in surrounding inflammation and fibrosis.

In no case did viable adenocarcinoma cells completely disappear after chemotherapy. It is

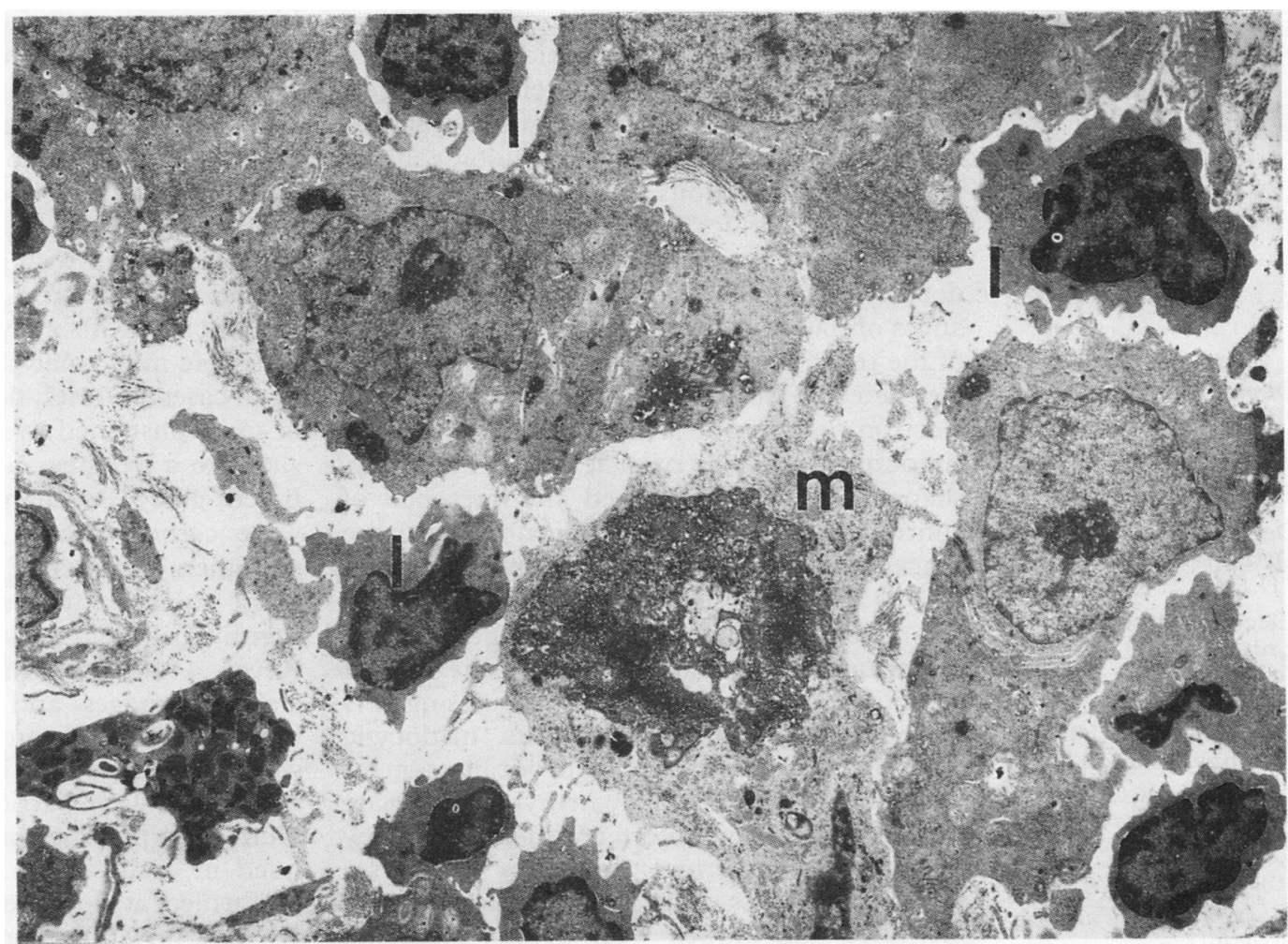

